\title{
Tabanidae (Insecta: Diptera) fauna of Kurıkkale province
}

\author{
Ferhat ALTUNSOY ${ }^{1 * 1}$, Bahriye AYAZ ${ }^{1}$ \\ ORCID: 0000-0003-1064-1541; 0000-0002-3967-1608 \\ ${ }^{1}$ Eskişehir Teknik Üniversitesi, Fen Fakültesi, Biyoloji Bölümü, 26470, Eskişehir, Turkey
}

\begin{abstract}
The article presents the horsefly fauna (Diptera: Tabanidae) of the Kırıkale province, Turkey. During the active periods in 2019 and 2020 a total of 2908 horseflies representing 40 species and classified in 3 subfamily. 36 species of these 40 samples are firstly recorded from Kırıkkale. The most common subfamily is Tabaninae represented with 34 species, followed by the subfamily Pangoninae-1 and Chrysopsinae- 5 species, respectively. Species collected in pastures, highlands, forestlands, rural and urban areas around Kırikkale province with Malaise and Nzi traps which were baited with 1-octen-3-ol. Worldwide distribution of the collected species is also given. Species except the Tabanus bromius Linnaeus, 1758, Haematopota kemali SZILADY, 1923, Tabanus spectabilis LOEW, 1858 and Tabanus regularis JAENNICKE, 1866 are firstly recorded from Kırıkkale.
\end{abstract}

Keywords: Diptera, Tabanidae, fauna, Kırıkkale, Turkey

\section{Kırıkale ili tabanidae (Insecta: Diptera) faunası}

\section{Özet}

Yapılan bu çalışmada, 2019-2020 yılları arasında Kırıkkale İli ve çevresinde bulunan Tabanidae üyeleri belirlenmiştir. Ergin birey yoğunluğunun yüksek olduğu bahar ve yaz aylarında, farklı yüksekliklerde bulunan mera ve otlaklara, dağlık ve ormanlık bölgelere, kırsal ve kentsel yerleşim bölgelerinin çevrelerine konuşlandırılan tuzaklar ile toplamda 2908 at sineği toplanmıştır. En yaygın alt familya olan Tabaninae alt familyasından 34, Pangoninae alt familyasından 1, Chrysopsinae alt familyasından 5 olmak üzere Tabanidae familyasına ait toplam 40 tür tespit edilmiştir. Tespit edilen bu 40 türün 36'sı Kırıkkale'den ilk kez bu çalışmayla bildirilmiştir. Bu türlerin toplandıkları noktalarla birlikte dünya üzerindeki dağılışları da verilmiştir. Tespit edilen türlerden Tabanus bromius LINNAEUS, 1758, Haematopota kemali SZILADY, 1923, Tabanus spectabilis LOEW, 1858 and Tabanus regularis JAENNICKE, 1866 dışındaki türler Kırıkkale İlinden ilk kez bildirilmiştir.

Anahtar kelimeler: Diptera, Tabanidae, Fauna, Kırıkkale, Türkiye

\section{Introduction}

Tabanidae family consist of dangerous species which impact on public health and sustainability of livestock due to transmitting viruses, bacteria, protozoans and helminths, which cause diseases in wild and domestic animals and humans $[8,10,12,22,31]$. Systematic researches on vector organisms provide understanding, predicting and controlling insect-borne diseases [6]. Although they are known as vector organisms worldwide, horseflies are also important regulators of ecosystems that have an essential role in pollination $[29,13]$.

Approximately 4300 species belonging to 137 genera of horse flies have been described worldwide [11]. Tabanidae represented 176 species and 15 subspecies in Turkey. The faunistic researches of Tabanidae in Turkey

\footnotetext{
${ }^{*}$ Corresponding author / Haberleşmeden sorumlu yazar: Tel.: +902223213550/4710; Fax.: +902223213550; E-mail: tabanidae@ msn.com

(c) Copyright 2021 by Biological Diversity and Conservation Geliş tarihi: 04.11.2020; Yayın tarihi: 15.04.2021 BioDiCon. 927-1120
} 
studied intensively for the last years which provide distribution and habitat demands of horseflies [1-5, 7, 17, 19- 21, 32].

The purpose of this study is to determine the distribution of Tabanidae family in the Kirıkale province which is located in the Central Anatolia and has extensive rural areas. Tabanidae fauna of Kırıkale was investigated for the first time with present study and 40 species belonging to Tabanidae family was reported.

\section{Materials and methods}

The study was conducted in the Kirikkale province of Turkey placed in Central Anatolia during active periods of horseflies from 2019-2020. Adult specimens collected with Malaise, Nzi and water traps at 32 different localities in pastures, highlands, forestlands, rural and urban areas around the Kirikkale (Fig.1). Malaise and Nzi traps are baited with 1-octen-3-ol. Collected specimens identified according to the keys [10, 27, 30]. Worldwide and Turkey distribution of each species is summarized according to $[9,10]$ and recent updates of Fauna Europaea, Global Species and the Catalogue of Life. Systema Dipterorum. Identified specimens are preserved in the Zoological Museum of Eskişehir Technical University.

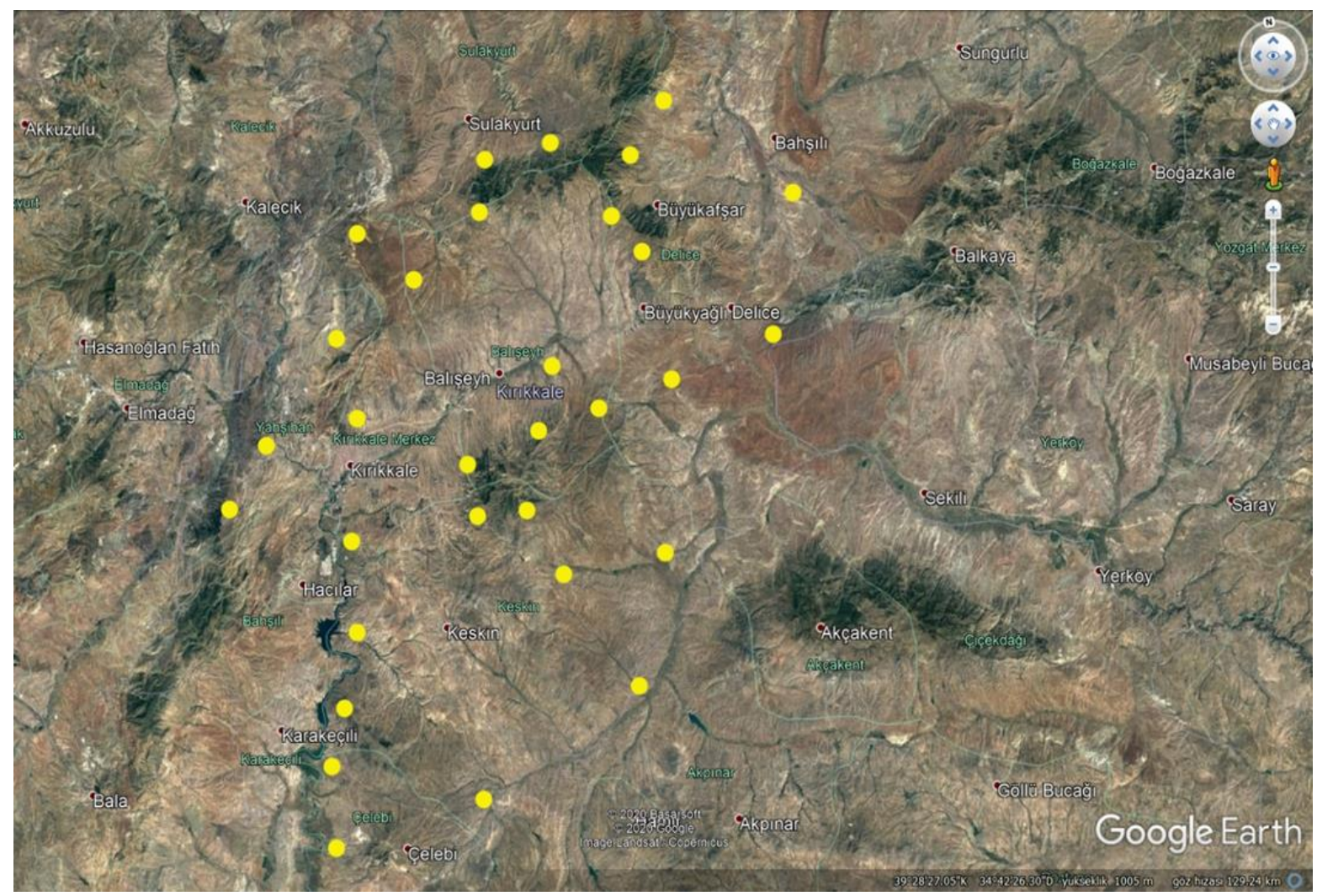

Figure 1: Collection area of horseflies

\section{Results}

Total 2908 horsefly species collected during the study at 32 different localities in Kurıkkale. All collected specimens classified into 40 species belonged to 3 subfamily. The most abundant species is Tabanus bromius with $\% 34,97$ and followed by Tabanus miki \%14,03; Tabanus bifarius \%6,74 (Table 1). The list of the determined horsefly species, including localities, dates, numbers, worldwide and Turkey distribution are presented below.

Alt Familya: PANGONINAE

Tribus: Pangoniini

1.Pangonius (s. str.) pyritosus (Loew, 1859)

Material examined: Keskin, 1397 m, 29.07.2019, 3 q $ᄋ, 1433$ m, 31.07.2019, 1 우; Delice, 1224 m, 09.07.2020, 1 우.

Distribution worldwide: Bulgaria, Greek Mainland, Hungary, Macedonia, Romania, South European Russia, Ukraine, West Russia

Distribution in Turkey: Amasya, Bayburt, Bursa, Çanakkale, Edirne, Erzincan, Erzurum, Eskişehir, Hakkâri, İstanbul, Kırklareli, Samsun.

Alt Familya: CHRYSOPSINAE

Tribus: Chrysopsini 
2. Chrysops (s. str.) caecutiens (Linnaeus, 1761)

Material examined: Keskin, 1556 m, 29.07.2019, 5 o $\circ$; 1410 m, 30.07.2019, 4 o $\bigcirc$; Delice, 1251 m, 03.08.2019, 1

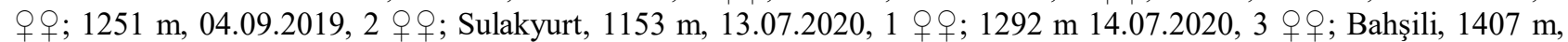
17.07.2019, 5 우; Çelebi, 1246 m, 27.08.2019, 1 우; 1427 m, 22.07.2020 4 우.

Distribution worldwide: Albania, Austria, Balearic Is., Belarus, Belgium, Bosnia and Herzegovina, Britain I., Bulgaria, Central European Russia, Croatia, Czech Republic, Danish mainland, Dodekánisos (Dodecanese Is.), East Palaearctic, Estonia, European Turkey, Finland, French mainland, Germany, Greek mainland, Hungary, Ireland, Italian mainland, Kikládes (Cyclades Is.), Kriti (Crete), Latvia, Liechtenstein, Lithuania, Luxembourg, Macedonia, Near East, North European Russia, Northwest European Russia, Norwegian mainland, Poland, Portuguese mainland, Romania, Sardinia, Sicily, Slovakia, Slovenia, South European Russia, Spanish mainland, Sweden, Switzerland, The Netherlands, Ukraine, Vóreion Aiyáion (North Aegean Is.), Former Yugoslavia.

Distribution in Turkey: Adıyaman, Afyon, Amasya, Ankara, Antalya, Artvin, Balıkesir, Bartın, Bayburt, Bingöl, Bolu, Burdur, Bursa, Çanakkale, Edirne, Erzurum, Eskişehir, Giresun, Gümüşhane, Hakkari, İçel, İstanbul, Karabük, Kars, Kastamonu, Kırklareli, Konya, Kütahya, Muğla, Muş, Nevşehir, Ordu, Rize, Samsun, Sinop, Sivas, Tekirdağ, Tokat, Trabzon, Uşak, Yalova, Zonguldak.

\section{Chrysops (s. str.) flavipes MEIGEN, 1804}

Material examined: Yahşihan, $1202 \mathrm{~m}, 14.08 .2019,3$ o $ᄋ$; Keskin, $1431 \mathrm{~m}, 18.09 .2019,5$ q ; $1563 \mathrm{~m}, 5$ 우; Sulakyurt, 14.07.2020, $1322 \mathrm{~m}, 3$ 우.

Distribution worldwide: Albania, Austria, Bulgaria, Croatia, Cyprus, Czech Republic, East Palaearctic, European Turkey, French mainland, Greek mainland, Hungary, Italian mainland, Macedonia, Near East, North Africa, Oriental region, Portuguese mainland, Romania, Sicily, Slovakia, South European Russia, Spanish mainland, Former Yugoslavia Distribution in Turkey: Adana, Adapazarı, Adıyaman, Ağrı, Afyon, Ankara, Antalya, Artvin, Balıkesir, Bayburt, Bilecik, Bolu, Bursa, Çanakkale, Çorum, Diyarbakır, Edirne, Elâzı̆̆g, Erzincan, Erzurum, Eskişehir, Hakkâri, Hatay, Iğdır, İçel, İstanbul, İzmir, Kastamonu, Karabük, Kars, Kırklareli, Konya, Kütahya, Manisa, Malatya, Muğla, Nevşehir, Niğde, Sinop, Sivas, Tekirdağ, Tokat, Trabzon, Uşak, Yozgat, Zonguldak.

\section{Chrysops (s. str.) italicus MEIGEN, 1804}

Material examined: Bahşili, $1271 \mathrm{~m}, 19.08 .2019,2$ o $ᄋ$; Delice, $1131 \mathrm{~m}, 04.09 .2019,1$ 우; $1209 \mathrm{~m}, 2$ o $ᄋ$; Çelebi, 21.07.2020, $1276 \mathrm{~m}, 4$ 우; 22.07.2020, $1443 \mathrm{~m}, 5$ 우.

Distribution worldwide: Albania, Bosnia and Herzegovina, Bulgaria, Corsica, Croatia, Cyprus, European Turkey, French mainland, Greek mainland, Hungary, Italian mainland, Macedonia, Near East, North Africa, Romania, Sardinia, Sicily, Slovenia, South European Russia, Spanish mainland, Ukraine, Former Yugoslavia.

Distribution in Turkey: Ankara, Antalya, Çanakkale, İzmir.

5. Chrysops (Petersenychrysops) buxtoni AUSTEN, 1922

Material examined: Keskin, $1254 \mathrm{~m}, 31.07 .2019,2$ q $\bigcirc ; 1624 \mathrm{~m}, 01.08 .2019,4 q \circ ; 1556 \mathrm{~m}, 08.07 .2020,4$ q 9 ; Karakeçili, 931 m, 23.08.2019, 1 우; Delice, 1052 m, 04.09.2019, 1 우; Sulakyurt, 14.07.2020, 1165 m, 2 우우 Balışeyh, 15.07.2020, 1298 m, 4 우; Keskin, 958 m, 25.08.2020, 1 우; 1625 m, 3 우.

Distribution worldwide: Israel, Iraq, Turkey

Distribution in Turkey: Ankara, Elazığ, Eskişehir, İçel, Kırşehir, Konya.

\section{Chrysops (Petersenychrysops) hamatus LOEW, 1858}

Material examined: Sulakyurt, 1050 m, 06.08.2019, 2 q o ; 1136 m, 21.09.2019, 3 q ; ; Karakeçili, 910 m, 26.08.2019,

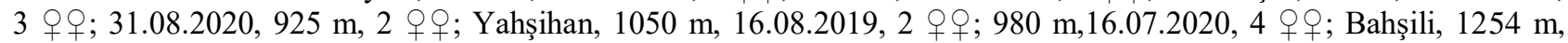
21.08.2019, 3 우; $1260 \mathrm{~m}, 19.07 .2020,5$ 우.

Distribution worldwide: Bulgaria, Dodekánisos (Dodecanese Is.), Near East, Turkey

Distribution in Turkey: Afyon, Ankara, Antalya, Çankırı, Denizli, Elazı̆̆, Eskişehir, İzmir, Muğla, Hatay.

Alt Familya: TABANINAE

Tribus: Tabanini

\section{Atylotus flavoguttatus (SZILADY, 1915)}

Material examined: Keskin, $1130 \mathrm{~m}, 31.08 .2019,3$ ㅇ; $1460 \mathrm{~m}, 2$ 2 $q ; 1342 \mathrm{~m}, 09.07 .2020,5$, 9 ; $1338 \mathrm{~m}$, 25.08.2020, 2 우; Delice, $1100 \mathrm{~m}, 04.08 .2019,4$ q $\circ ; 1278 \mathrm{~m}, 05.08 .2019,2$ 우; $980 \mathrm{~m}, 04.09 .2019,2$ o $ᄋ ; 900 \mathrm{~m}$, 20.09.2019, 1 우; $1180 \mathrm{~m}, 11.07 .2020,5$ 우; $1046 \mathrm{~m}, 26.08 .2020,1$ 우; Balışeyh, $975 \mathrm{~m}, 12.08 .2019,2$ 우; 1315 $\mathrm{m}, 13.08 .2019,1$ 우; $1285 \mathrm{~m}, 15.07 .2020,4$ 우; $1295 \mathrm{~m}, 28.08 .2020,1$ 우; Çelebi, $1426 \mathrm{~m}, 21.07 .2020,3$ 우; 1449 $\mathrm{m}, 22.07 .2020,2$ 우우.

Distribution worldwide: Austria, Croatia, European Turkey, French mainland, Hungary, Moldova, Near East, North Africa, Portuguese mainland, Romania, South European Russia, Spanish mainland, Ukraine 
Distribution in Turkey: Afyon, Ankara, Balıkesir, Çanakkale, Edirne, Eskişehir, İstanbul, Kırklareli, Kırşehir, Konya, Nevşehir, Tekirdağ, Yozgat.

\section{Atylotus hendrixi LECLERCQ, 1967}

Material examined: Keskin, 1682 m, 30.07.2019, 8 q $; 1710$ m, 01.08.2019, 6 + $;$; 1486 m, 18.09.2019, 3 $\circ ; 1547$ $\mathrm{m}, 08.07 .2020,11$ 우; 1710 m, 09.07.2020, 7 우; Balışeyh, 1325 m, 12.08.2019, 5 o $\bigcirc ; 1449 \mathrm{~m}, 15.07 .2020,8$ 우; $1690 \mathrm{~m}, 28.08 .2020,3$ 우; Sulakyurt, $1207 \mathrm{~m}, 07.08 .2019,6$ 우; $1318 \mathrm{~m}, 09.08 .2019,3$ 우; $1320 \mathrm{~m}, 14.07 .2020,8$ 우.

Distribution worldwide: Turkey, Iran.

Distribution in Turkey: Adana, Afyon, Ankara, Artvin, Burdur, Erzurum, Eskişehir, Hakkari, Kayseri, Konya, Trabzon, Van, Yozgat.

\section{Atylotus rusticus (LINNAEUS, 1761)}

Material examined: Delice, 1290 m, 04.08.2019, 2 q ; Keskin, 1687 m, 09.07.2020, 3 q ; 1701 m, 25.08.2020, 1 qㅇ.

Distribution worldwide: Austria, Belgium, Bosnia and Herzegovina, Britain I., Bulgaria, Central European Russia, Croatia, Czech Republic, Danish mainland, East Palaearctic, Estonia, European Turkey, Finland, French mainland, Germany, Greek mainland, Hungary, Italian mainland, Lithuania, Luxembourg, North Africa, Northwest European Russia, Norwegian mainland, Poland, Romania, Sicily, Slovakia, Slovenia, South European Russia, Spanish mainland, Sweden, Switzerland, The Netherlands, Ukraine, Former Yugoslavia.

Distribution in Turkey: Ankara, Eskişehir, Trakya Bölgesi.

\section{Therioplectes tricolor ZELLER, 1842}

Material examined: Bahşili, 1529 m, 20.08.2019, 1 qo ; Keskin, 1695 m, 18.09.2019, 2 q ; Balışeyh, 1458 m, 15.07.2020, 4 우; $1650 \mathrm{~m}, 28.07 .2020,3$ q 9.

Distribution worldwide: Bulgaria, European Turkey, Greek mainland, Italian mainland, Near East, Romania, Sicily, South European Russia, Vóreion Aiyáion (North Aegean Is.)

Distribution in Turkey: Aksaray, Amasya, Ankara, Artvin, Bayburt, Bilecik, Bursa, Erzurum, Eskişehir, Gümüşhane. İzmir, Kayseri, Kütahya, Muğla, Muğla, Nevşehir, Niğde, Tekirdağ, Uşak, Yozgat.

\section{Hybomitra caucasi (SZILADY, 1923)}

Material examined: Yahşihan, 1116 m, 14.08.2019, 2 q ; Karakeçili, 911 m, 20.07.2020, 3 우.

Distribution worldwide: European Turkey, Near East, Romania, Russia

Distribution in Turkey: Ankara, Çanakkale, Erzurum, Hakkari, İstanbul, Kırklareli, Kuzey Anadolu Dağları, Tekirdağ.

\section{Tabanus autumnalis LINNEAUS, 1761}

Material examined: Delice, $1148 \mathrm{~m}, 04.08 .2019,4$ + $q ; 1346 \mathrm{~m}, 05.09 .2019,3$ q $q ; 1266 \mathrm{~m}, 11.07 .2020,6 q \circ$; Balışeyh, $1535 \mathrm{~m}, 15.07 .2010,1$ o $ᄋ$; Sulakyurt, $1264 \mathrm{~m}, 08.08 .2019,3$ q ; $1324 \mathrm{~m}, 02.09 .2019,5$ 우; $1333 \mathrm{~m}$, 13.07.2020, 9 우; $1225 \mathrm{~m}, 14.07 .2020,5$ 우; 1243 m, 27.08.2020, 2 q ; ; Keskin, 1085 m, 31.07.2019, 7 우; 1312 $\mathrm{m}, 01.08 .2019,2$ 우; $1655 \mathrm{~m}, 08.07 .2020,8$ 우; Çelebi, $1348 \mathrm{~m}, 29.08 .2019,2$ 우; $1450 \mathrm{~m}, 22.07 .2020,4$ 우; 1236 m, 01.09.2020, 1 우.

Distribution worldwide: Albania, Austria, Balearic Is., Belgium, Bosnia and Herzegovina, Britain I., Bulgaria, Central European Russia, Corsica, Croatia, Cyprus, Czech Republic, Danish mainland, East European Russia, East Palaearctic, Estonia, European Turkey, French mainland, Germany, Greek mainland, Hungary, Italian mainland, Lithuania, Luxembourg, Macedonia, Malta, Near East, North Africa, Northwest European Russia, Norwegian mainland, Poland, Portuguese mainland, Romania, Sardinia, Sicily, Slovakia, Slovenia, South European Russia, Spanish mainland, Sweden, Switzerland, The Netherlands, Ukraine, Former Yugoslavia.

Distribution in Turkey: Adana, Afyon, Aksaray, Amasya, Ankara, Artvin, Balıkesir, Bayburt, Bilecik, Bolu, Burdur, Bursa, Çanakkale, Çankırı, Çorum, Denizli, Diyarbakır, Edirne, Elazı̆̆g, Erzincan, Erzurum, Eskişehir, Giresun, Hatay, Iğdır, İstanbul, İzmir, Kars, Kastamonu, Kırklareli, Konya, Kütahya, Malatya, Muğla, Nevşehir, Niğde, Ordu, Sinop, Tekirdağ, Trabzon, Tunceli, Uşak, Van, Yozgat, Zonguldak.

\section{Tabanus bifarius LOEW, 1858}

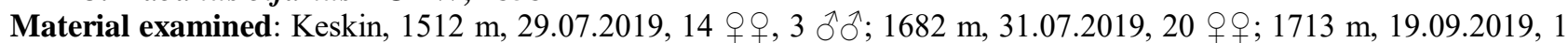
우우 $1426 \mathrm{~m}, 08.07 .2020,11$ 우, 3 ふึ了; $1566 \mathrm{~m}, 25.08 .2020,4$ 우; Delice, $1035 \mathrm{~m}, 04.08 .2019,1$ 우; $1311 \mathrm{~m}$, 12.07.2020, 4 q , , Sulakyurt, $1064 \mathrm{~m}, 07.08 .2019,2$ q $ᄋ ; 1249 \mathrm{~m}, 08.08 .2019,6$ 우; $1257 \mathrm{~m}, 13.07 .2020,4$ 우; 1322 $\mathrm{m}, 14.07 .2020,4$ 우; $1305 \mathrm{~m}, 27.08 .20202$ 우; Balışeyh, $1215 \mathrm{~m}, 10.08 .2019,1$ 우; $1385 \mathrm{~m}, 11.08 .2019,7 q$ 우, 3

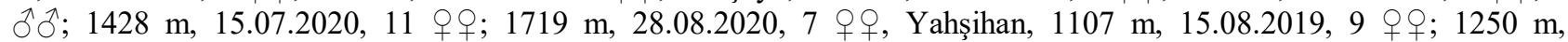

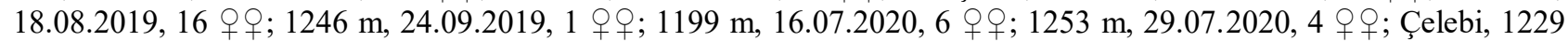
$\mathrm{m}, 28.08 .2019,9$ 우; $1442 \mathrm{~m}, 30.08 .2019,4$ 우; $1356 \mathrm{~m}, 28.09 .2019,1$ 우; Bahşili, $1255 \mathrm{~m}, 20.08 .2019,8$ 우; 
$1539 \mathrm{~m}, 26.08 .2019,2$ 우; $1322 \mathrm{~m}, 17.07 .2020,5$ 우, 1 ふَ̋; $1487 \mathrm{~m}, 19.07 .2020,6$ 우; $1520 \mathrm{~m}, 30.08 .2020,13$ 우, $3 \hat{o}$.

Distribution worldwide: Albania, Austria, Bulgaria, Croatia, European Turkey, French mainland, Germany, Greek mainland, Hungary, Italian mainland, Macedonia, Moldova, Near East, North Africa, Romania, Slovakia, Slovenia, South European Russia, Spanish mainland, Ukraine, Former Yugoslavia.

Distribution in Turkey: Adana, Afyon, Balıkesir, Bartın, Bayburt, Bilecik, Bolu, Bursa, Çanakkale, Çorum, Edirne, Erzurum, Eskişehir, Gümüşhane, İçel, İstanbul, İzmir, İzmit, Kahramanmaraş, Kars, Kastamonu, Kayseri, Kırklareli, Kırşehir, Konya, Mardin, Muğla, Nevşehir, Niğde, Samsun, Sinop, Sivas, Tekirdağ, Yalova, Yozgat.

\section{Tabanus briani LECLERCQ, 1962}

Material examined: Çelebi, 1339 m, 28.08.2019, 1 qo ; Karakeçili, 918 m, 20.07.2020, 2 q ; Bahşili, 1122 m, 21.08.2019, 1 우, $1518 \mathrm{~m}, 19.07 .2020,4$ 우.

Distribution worldwide: Bosnia and Herzegovina, Bulgaria, Croatia, European Turkey, French mainland, Italian mainland, Sicily, Slovakia, Slovenia, Spanish mainland

Distribution in Turkey: Ankara, Balıkesir, Bartın, Bolu, Bursa, Çanakkale, Düzce, , Karabük, Kastamonu, Kırklareli, Kütahya, Sinop, Tekirdağ, Zonguldak.

\section{Tabanus bromius LINNAEUS, 1758}

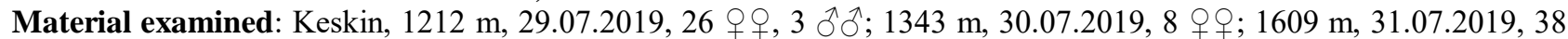

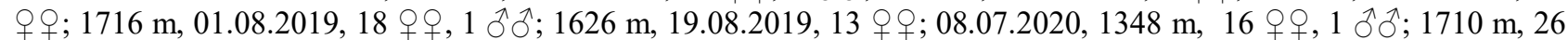

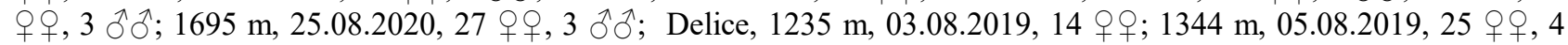

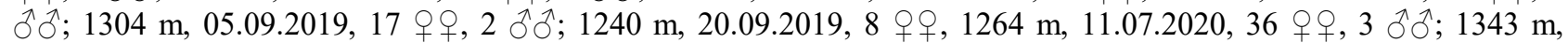
26.08.2020, 21 우; Sulakyurt, $1064 \mathrm{~m}, 06.08 .2019,20$ 우; $1294 \mathrm{~m}, 09.08 .2019,17$ 우; $1143 \mathrm{~m}, 01.09 .2019,9$ 우;

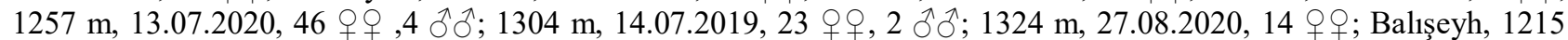

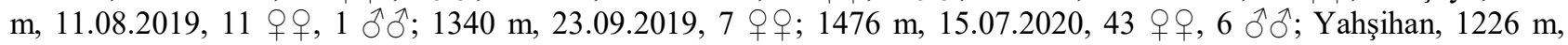

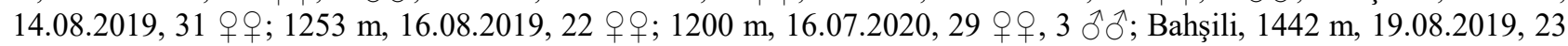

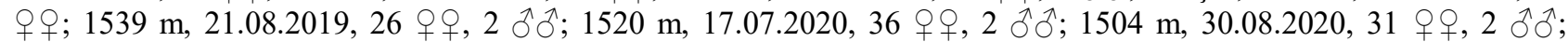
Karakeçili, 890 m, 23.08.2019, 33 우, 3 ôㅎㄱ 930 m, 26.08.2019, 19 우; 846 m, 27.09.2019, 8 우; 900 m,

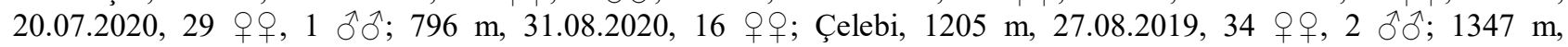

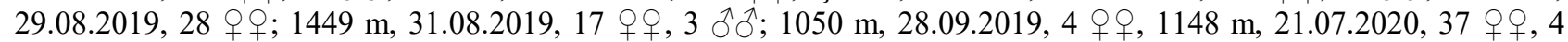

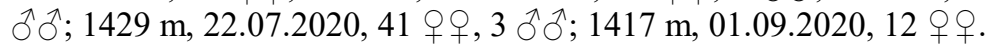

Distribution worldwide: Albania, Austria, Belgium, Bosnia and Herzegovina, Britain I., Bulgaria, Central European Russia, Croatia, Czech Republic, Danish mainland, East European, Russia, East Palaearctic, Estonia, European Turkey, Finland, French mainland, Germany, Greek mainland, Hungary, Ireland, Italian mainland, Latvia, Liechtenstein, Lithuania, Luxembourg, Macedonia, Moldova, Near East, North Africa, North European Russia, Northwest European Russia, Norwegian mainland, Poland, Portuguese mainland, Romania, Sardinia, Sicily, Slovakia, Slovenia, South European Russia, Spanish mainland, Sweden, Switzerland, The Netherlands, Ukraine, Former Yugoslavia.

Distribution in Turkey: Adana, Adıyaman, Afyon, Ağrı, Aksaray, Amasya, Ankara, Antalya, Artvin, Aydın, Balıkesir, Bartın, Bayburt, Bilecik, Bitlis, Bolu, Bursa, Çankırı, Çorum, Denizli, Diyarbakır, Edirne, Elazı̆̆, Erzurum, Eskişehir, Giresun, Gümüşhane, Hakkari, Hatay, Isparta, İçel, İzmir, Kahramanmaraş, Kars, Kayseri, Kırıkkale, Kırklareli, Kırşehir, Konya, Kütahya, Malatya, Muğla, Muş, Nevşehir, Niğde, Ordu, Rize, Sakarya, Sakarya, Samsun, Siirt, Sinop, Sivas, Tekirdağ, Tokat, Trabzon, Tunceli, Uşak, Van, Yalova.

\section{Tabanus cordiger MEIGEN, 1820}

Material examined: Keskin, 1650 m, 29.07.2019, 2 우; 1478 m, 18.08.2019, 4 우; 1390 m, 25.08.2020, 5 우; Çelebi, $1312 \mathrm{~m}, 28.08 .2019,2$ o $\odot ; 1287 \mathrm{~m}, 21.07 .2020,1$ q ; $1400 \mathrm{~m}, 01.09 .2020,1$ o ; Bahşili, $1150 \mathrm{~m}$, 20.08.2019, 4 우; $1532 \mathrm{~m}, 22.08 .2019,2$ 우; 1148 m, 18.07.2020, 1 웅 Balışeyh, 1714 m, 28.08.2020, 3 우오.

Distribution worldwide: Albania, Austria, Belgium, Bosnia and Herzegovina, Britain I., Bulgaria, Canary Is., Central European Russia, Croatia, Czech Republic, East European Russia, European Turkey, Finland, French mainland, Germany, Greek mainland, Hungary, Italian mainland, Lithuania, Macedonia, Near East, North Africa, Northwest European Russia, Poland, Portuguese mainland, Romania, Slovakia, Slovenia, South European Russia, Spanish mainland, Sweden, Switzerland, The Netherlands, Ukraine, Former Yugoslavia.

Distribution in Turkey: Adana, Afyon, Ankara, Balıkesir, Bilecik, Bolu, Bursa, Elazı̆̆, Erzurum, Eskişehir, İçel, Kırklareli, Kütahya, Yozgat.

\section{Tabanus eggeri SCHİNERİ, 1868}

Material examined: Sulakyurt, 1235 m, 13.07.2020, 3 q ; Y Yahşihan, 1252 m, 16.08.2019, 2 ○ ; 1080 m, 16.07.2020, 4 우; 1236 m, 29.08.2020, 1 우; Karakeçili, 930 m, 20.07.2020, 2 우.

Distribution worldwide: Albania, Bulgaria, Croatia, European Turkey, French mainland, Italian mainland, Near East, North Africa, Portuguese mainland, Spanish mainland, Former Yugoslavia. 
Distribution in Turkey: Adana, Adapazarı, Afyon, Ankara, Antalya, Balıkesir, Bartın, Bilecik, Bolu, Bursa, Çanakkale, Edirne, Eskişehir, Hakkari, İçel, İstanbul, İzmit, Kırklareli, Konya. Kütahya, Nevşehir, Niğde, Sinop, Tekirdağ, Yalova, Yozgat.

\section{Tabanus exlusus PANDELLE, 1883}

Material examined: Keskin, 1249 m, 01.08.2019, 4 q ; 1387 m, 18.09.2019, 1 q ; 1310 m, 08.07.2020, 5 q $\bigcirc$; Delice, 1105 m, 04.09.2019, 1 우; 1250 m, 26.08.2020, 4 우; Balışeyh, $1264 \mathrm{~m}, 15.07 .2020,3$ 우; $1685 \mathrm{~m}$, 28.08.2020, 1 우.

Distribution worldwide: Albania, Bosnia and Herzegovina, Bulgaria, Croatia, European Turkey, French mainland, Greek mainland, Hungary, Italian mainland, Macedonia, Moldova, Near East, Portuguese mainland, Romania, Slovakia, Spanish mainland, Former Yugoslavia.

Distribution in Turkey: Ankara, Antalya, Aydın, Balıkesir, Bilecik, Bolu. Bursa, Çanakkale, Denizli, Edirne, Erzurum, Eskişehir, Hakkari, İçel, İzmir, Kayseri, Kırklareli, Konya, Konya, Kütahya, Muğla, Nevşehir, Samsun, Sinop, Tekirdă̆, Uşak, Yalova.

\section{Tabanus fraseri AUSTEN, 1925}

Material examined: Sulakyurt, 1253 m, 02.09.2019, 1 q ; 1319 m, 21.09.2019, 1 q ; Karakeçili, 896 m, 23.08.2019, 2 우; Çelebi, $1269 \mathrm{~m}, 30.08 .2019,2$ 우; $1204 \mathrm{~m}, 01.09 .2020,1$ 우.

Distribution worldwide: Albania, Bulgaria, Croatia, European Turkey, Greek mainland, Near East

Distribution in Turkey: Ankara, Aydın, Balıkesir, Bilecik, Bursa, Düzce, Edirne, Eskişehir, İstanbul, İzmir, Karabük, Kastamonu, Kırklareli, Kütahya, Muğla, Tekirdağ Uşak.

\section{Tabanus glaucopis MEIGEN, 1820}

Material examined: Keskin, 1689 m, 31.08.2019, 2 q o ; 928 m, 18.09.2019, 1 q $\circ ; 1700$ m, 09.07.2020, 8 q ; Delice, $1110 \mathrm{~m}, 04.09 .2019$, 1 우; $1315 \mathrm{~m}, 26.08 .2020,4$ 우; Yahşihan, $1096 \mathrm{~m}, 15.08 .2019,4$ 우; $1248 \mathrm{~m}$, 16.08.2019, 5 우; Karakeçili, 860 m, 31.08.2020, 2 우.

Distribution worldwide: Albania, Austria, Belgium, Bosnia and Herzegovina, Britain I., Bulgaria, Central European Russia, Croatia, Czech Republic, East Palaearctic, European Turkey, French mainland, Germany, Greek mainland, Hungary, Italian mainland, Lithuania, Macedonia, Near East, Northwest European Russia, Norwegian mainland, Poland, Portuguese mainland, Romania, Sardinia, Sicily, Slovakia, Slovenia, South European Russia, Spanish mainland, Sweden, Switzerland, Ukraine, Former Yugoslavia.

Distribution in Turkey: Adapazarı, Afyon, Ankara, Artvin, Aydın, Balıkesir, Bilecik, Bolu, Bursa, Çanakkale, Çorum, Denizli, Düzce, Edirne, Erzurum, Eskişehir, Hakkari, İçel, İstanbul, İzmir, Karabük, Kastamonu, Kırklareli, Konya. Kütahya, Muş, Niğde, Rize, Samsun, Sinop, Tekirdağ, Tokat, Trabzon, Uşak, Van.

\section{Tabanus latetinctus AUSTEN, 1920}

Material examined: Delice, 1348 m, 03.08.2019, 2 o $q$; 1028 m, 11.07.2020, 4 q ; ; Balışeyh, 1448 m, $11.08 .2019,1$ 우; $1710 \mathrm{~m}, 15.07 .2020,5$ q $ᄋ$; Bahşili, $1322 \mathrm{~m}, 18.07 .2020,3$ 우.

Distribution worldwide: Turkey, Iran, Afghanistan, Central Asia, Caucasia

Distribution in Turkey: Adana, Ankara, Artvin, Bayburt, Bilecik, Çanakkale, Çankırı, Çorum, Elazı̆̆, Erzurum, Eskişehir, Gaziantep, Gümüşhane, Hakkari, İzmir, Kayseri, Konya, Kütahya, Nevşehir, Niğde, Siirt, Sivas, Tekirdağ, Yozgat.

\section{Tabanus lunatus FABRICIUS, 1794}

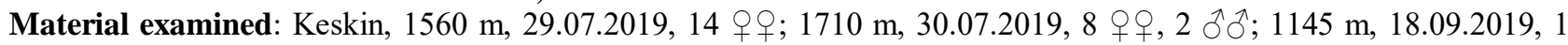

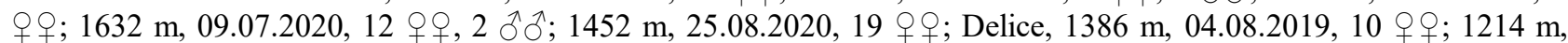

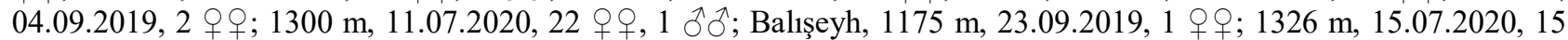

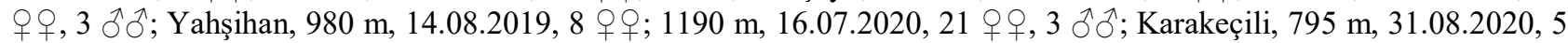

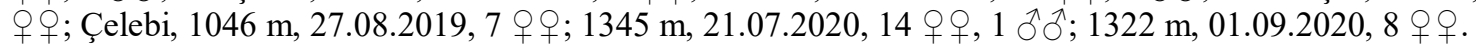

Distribution worldwide: Albania, Bosnia and Herzegovina, Bulgaria, Croatia, East Palaearctic, European Turkey, French mainland, Greek mainland, Italian mainland, Near East, North Africa, Portuguese mainland, Romania, Slovenia, Spanish mainland, Former Yugoslavia.

Distribution in Turkey: Afyon, Amasya, Ankara, Antalya, Aydın, Balıkesir, Bilecik, Bolu, Bursa, Çanakkale, Edirne, Erzurum, Eskişehir, Gümüşhane, Hakkari, Hatay, İçel, İstanbul, İzmir, İzmit, Karabük, Kars, Kastamonu, Kayseri, Kırklareli, Konya, Kütahya, Manisa, Mardin, Muğla, Samsun, Sinop, Tekirdağ, Tokat, Uşak, Yalova.

\section{Tabanus martinii KRÖBER, 1928}

Material examined: Delice, $1178 \mathrm{~m}, 04.09 .2019,4$ q $\circ$; $1315 \mathrm{~m}, 26.08 .2020,3$ 우; Yahşihan, $1235 \mathrm{~m}, 16.08 .2019,2$ 우우 $1049 \mathrm{~m}, 24.09 .2019,3$ 우; $1251 \mathrm{~m}, 29.08 .2020,1$ 우; Bahşili, $1352 \mathrm{~m}, 19.08 .2019,2$ 우; $1145 \mathrm{~m}, 26.09 .2019$, 3 우우 Çelebi, $1426 \mathrm{~m}, 31.08 .2019,2$ 우웅 $1445 \mathrm{~m}, 22.07 .2020,1$ 우. 
Distribution worldwide: European Turkey, Near East, Romania, Spanish mainland

Distribution in Turkey: Afyon, Ankara, Aydın, Edirne, Eskişehir, Hakkari, Kırklareli Konya, Kütahya, Tekirdağ.

\section{Tabanus miki BRAUER, 1880}

Material examined: Keskin, $1314 \mathrm{~m}, 30.07 .2019,7$ q ; $1694 \mathrm{~m}, 01.08 .2019,11$ q ${ }^{\prime} ; 1544 \mathrm{~m}, 08.07 .2020,19$ q $\circ$; 1478 m, 25.08.2020, 17 우; Delice, 1158 m, 03.08.2019, 6 우; 1338 m, 05.08.2019, 5 o ; $1087 \mathrm{~m}, 04.09 .2019,3$ 우우 $1344 \mathrm{~m}, 10.07 .2020,17$ 우; $1088 \mathrm{~m}, 11.07 .2020,29$ 우; $1255 \mathrm{~m}, 26.08 .2020,3$ 우; Sulakyurt, $974 \mathrm{~m}$, 06.08.2019, 4 우; $1312 \mathrm{~m}, 09.08 .2019,16$ 우; $1233 \mathrm{~m}, 03.09 .2019,1$ 우; $1024 \mathrm{~m}, 13.07 .2020,23$ 우; $1237 \mathrm{~m}$, 27.08.2020, 7 우; Balışeyh, 1075 m, 11.08.2019, 11 우; 1319 m, 13.08.2019, 18 우; $1581 \mathrm{~m}, 15.07 .2020,27$ 우; $1711 \mathrm{~m}, 28.07 .2020,3$ 우; Yahşihan, $1215 \mathrm{~m}, 15.08 .2019,14$ 우; $1252 \mathrm{~m}, 18.08 .2019,22$ q $\bigcirc ; 900 \mathrm{~m}, 24.09 .2019,1$ 우; $1246 \mathrm{~m}, 16.07 .2020,24$ 우 ; Bahşili, $1322 \mathrm{~m}, 20.08 .2019,9$ q ; $1539 \mathrm{~m}, 22.08 .2019,17$ q $9 ; 1447 \mathrm{~m}$, 18.07.2020, 29 우; 1429 m, 30.08.2020, 3 우우 Karakeçili, 790 m, 23.08.2019, 2 우; 931 m, 20.07.2020, 9 오; Çelebi, $1096 \mathrm{~m}, 28.08 .2019,6$ 우; $1416 \mathrm{~m}, 31.08 .2019,9$ 우; $1210 \mathrm{~m}, 21.07 .2020,15$ 우; $1422 \mathrm{~m}, 22.07 .2020,21$ 우.

Distribution worldwide: Albania, Austria, Belgium, Britain I., Bulgaria, Central European Russia, Croatia, Czech Republic, Danish mainland, East European Russia, East Palaearctic, European Turkey, French mainland, Germany, Greek mainland, Hungary, Italian mainland, Lithuania, Macedonia, Near East, North European Russia, Northwest European Russia, Poland, Portuguese mainland, Romania, Slovakia, Slovenia, South European Russia, Spanish mainland, Sweden, Ukraine

Distribution in Turkey: Ankara, Artvin, Balıkesir, Bartın, Bayburt, Bilecik, Bolu, Bursa, Çanakkale, Denizli, Düzce, Elazığ, Erzurum, Eskişehir, Giresun, Hakkari, İçel, İstanbul, İstanbul, İzmir, Kars, Kastamonu, Kayseri, Kırklareli, Konya, Kütahya, Manisa, Muğla, Muğla, Nevşehir, Ordu, Samsun, Tokat, Trabzon, Uşak, Yozgat, Zonguldak.

\section{Tabanus oppugnator AUSTEN, 1925}

Material examined: Keskin, 1710 m, 29.07.2019, 1 q o ; 1680 m, 08.07.2020, 3 q ; ; Balışeyh, 1710 m, $15.07 .2020,2$ 우우 B Bahşili, $1325 \mathrm{~m}, 20.08 .2019,1$ 우웅 $1539 \mathrm{~m}, 18.07 .2020,2$ 우우.

Distribution worldwide: Turkey, Iran

Distribution in Turkey: Balıkesir, Bolu, Çanakkale, Çankırı, Elazı̆̆g, Kastamonu, Kütahya, Sinop.

\section{Tabanus quatuornatatus MEIGEN, 1820}

Material examined: Keskin, 1335 m, 29.07.2019, 2 우; 1513 m, 08.07.2020, 6 우; 1700 m, 25.08.2020, 1 우; Delice, $1000 \mathrm{~m}, 03.08 .2019,2$ o $; ; 1317 \mathrm{~m}, 05.08 .2019,1$ o $; 1340 \mathrm{~m}, 11.07 .2020,5$ q ; Çelebi, $1196 \mathrm{~m}$, 27.08.2019, 1 ㅇ; $1426 \mathrm{~m}, 30.08 .2019,2$ 우; $1397 \mathrm{~m}, 21.07 .2020,4$ q ; $1215 \mathrm{~m}, 22.07 .2020,4$ 우.

Distribution worldwide: Albania, Austria, Belgium, Bosnia and Herzegovina, Britain I., Bulgaria, Croatia, Czech Republic, Danish Mainland, East Palaearctic, European Turkey, French mainland, Germany, Greek mainland, Hungary, Italian mainland, Macedonia, Moldova, Near East, North Africa, Poland, Portuguese mainland, Romania, Slovakia, Slovenia, South European Russia, Spanish mainland, Switzerland, Ukraine, Former Yugoslavia.

Distribution in Turkey: Adana, Amasya, Ankara, Artvin, Aydın, Balıkesir, Bayburt, Bilecik, Bolu, Bursa, Çanakkale, Çorum, Denizli, Erzurum, Eskişehir, Giresun, Gümüşhane, Hakkari, Hatay, İçel, İzmir, Kastamonu, Kırklareli, Konya, Kütahya, Muğla, Niğde, Sinop, Tekirdağ, Trabzon, Uşak, Yozgat.

\section{Tabanus regularis JAENNICKE, 1866}

Material examined: Keskin, 1710 m, 29.07.2019, 2 우; 1680 m, 08.07.2020, 1 우; 1713 m, 25.08.2020, 2 우; Balışeyh, $1581 \mathrm{~m}, 12.08 .2019,3$ q $ᄋ$; $1315 \mathrm{~m}, 13.08 .2019,2$ q $ᄋ$; $1710 \mathrm{~m}, 15.07 .2020,1$ 우.

Distribution worldwide: Bosnia and Herzegovina, Bulgaria, Corsica, Croatia, Cyprus, European Turkey, French mainland, Greek mainland, Hungary, Italian mainland, Macedonia, Malta, Near East, North Africa, Portuguese mainland, Romania, Sicily, Slovakia, South European Russia, Spanish mainland

Distribution in Turkey: Afyon, Ankara, Antalya, Aydın, Balıkesir, Bilecik, Bursa, Çanakkale, Denizli, Edirne, Elazığ, Erzurum, Eskişehir, Hakkari, İçel, İstanbul, İzmir, Kırklareli, Konya, Kütahya, Muğla, Tekirdağ, Yozgat.

\section{Tabanus spectabilis LOEW, 1858}

Material examined: Sulakyurt, 1090 m, 06.08.2019, 1 q ; ; Karakeçili, 931 m, 20.07.2020, 1 ㅇ.

Distribution worldwide: Albania, Bulgaria, Croatia, European Turkey, French mainland, Greek mainland, Hungary, Italian mainland, Macedonia, Moldova, Near East, North Africa, Romania, Sicily, Slovenia, South European Russia, Spanish mainland, Former Yugoslavia.

Distribution in Turkey: Ankara, Bilecik, Bolu, Çanakkale, Çankırı, Çorum, Edirne, Elazı̆̆, Erzincan, Erzurum, Eskişehir, Giresun, İçel, Kayseri, Kırklareli, Konya, Niğde, Tekirdağ, Yozgat. 


\section{Tabanus spodopterus MEIGEN, 1820}

Material examined: Keskin, 1235 m, 30.07.2019, 4 q ; 1437 m, 01.08.2019, 1 q ; 1355 m, 08.07.2020, 7 q ; Delice, 1278 m, 04.08.2019, 2 우; 1349 m, 11.07.2020, 5 우; Sulakyurt, $1214 \mathrm{~m}, 07.08 .2019,3$, 9 ; $1324 \mathrm{~m}$, 14.07.2020, 4 우; 1255 m, 27.08.2020, 1 우; Yahşihan, 1015 m, 14.08.2019, 1 우; 1252 m, 16.07.2020, 4 우; Karakeçili, $931 \mathrm{~m}, 20.07 .2020,2$ 우.

Distribution worldwide: Austria, Bosnia and Herzegovina, Bulgaria, Croatia, Czech Republic, European Turkey, French mainland, Germany, Greek mainland, Hungary, Italian mainland, Macedonia, Moldova, Near East, Poland, Portuguese mainland, Romania, Slovakia, South European Russia, Spanish mainland, Switzerland

Distribution in Turkey: Adapazarı, Afyon, Amasya, Ankara, Antalya, Artvin, Balıkesir, Bolu, Bursa, Çanakkale, Çorum, Denizli, Edirne, Erzurum, Eskişehir, İçel, İstanbul, Karabük, Kars, Kastamonu, Kırklareli, Konya, Kütahya, Muğla, Tekirdağ, Trabzon, Uşak, Yalova, Zonguldak.

\section{Tabanus tergestinus EGGER, 1859}

Material examined: Keskin, 1266 m, 30.07.2019, 11 우; 1655 m, 01.08.2019, 13 우; 1530 m, 09.07.2020, 14 우; Delice, $1349 \mathrm{~m}, 03.08 .2019,9$ 우; $1066 \mathrm{~m}, 04.09 .2019,1$ 우; $1340 \mathrm{~m}, 12.07 .2020,7$ 우; $1278 \mathrm{~m}, 26.08 .2020,6$ 우; Sulakyurt, $1050 \mathrm{~m}, 06.08 .2019,8$ 우; $1244 \mathrm{~m}, 07.08 .2019,10$ 우; $1024 \mathrm{~m}, 02.09 .2019,2$ q $;$; $1388 \mathrm{~m}, 13.07 .2020$,

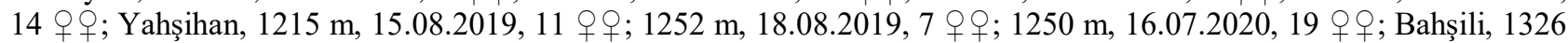

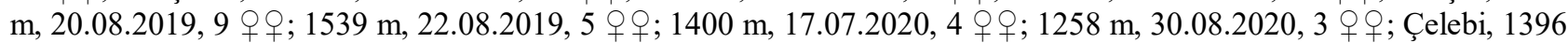
$\mathrm{m}, 27.08 .2019,3$ 우; $1449 \mathrm{~m}, 22.07 .2020,11$ 우; $1226 \mathrm{~m}, 01.09 .2020,1$ 웅.

Distribution worldwide: Albania, Austria, Bosnia and Herzegovina, Bulgaria, Central European Russia, Croatia, Czech Republic, European Turkey, French mainland, Germany, Greek mainland, Hungary, Italian mainland, Iran, Macedonia, Moldova, Near East, Poland, Romania, Slovakia, Slovenia, South European Russia, Spanish mainland, Switzerland, Ukraine, Former Yugoslavia.

Distribution in Turkey: Adapazarı, Afyon, Ankara, Antalya, Artvin, Artvin, Aydın, Balıkesir, Bartın, Bolu, Bursa, Edirne, Elazı̆̆ Erzurum, Gümüşhane, Hatay, İçel, İstanbul, Kastamonu, Kırklareli, Muğla, Ordu, Samsun, Sinop, Tekirdağ, Yalova, Zonguldak.

\section{Tabanus unifasciatus LOEW, 1858}

Material examined: Keskin, $1680 \mathrm{~m}, 29.07 .2019,5$ q $q ; 1700 \mathrm{~m}, 08.07 .2020,9$ q $q ; 1550 \mathrm{~m}, 09.07 .2020,4$ q $ᄋ$; Bahşili, $1522 \mathrm{~m}, 19.08 .2019,1$ qo ; $1510 \mathrm{~m}, 17.07 .2020,9$ o $9 ; 1490 \mathrm{~m}, 18.07 .2020,7$ o $ᄋ$; Çelebi, $1449 \mathrm{~m}$, 21.07.2020, 3 우우; $1350 \mathrm{~m}, 22.07 .2020,2$ 우오.

Distribution worldwide: Albania, Austria, Bosnia and Herzegovina, Bulgaria, Croatia, European Turkey, French mainland, Greek mainland, Hungary, Italian mainland, Macedonia, Moldova, Near East, North Africa, Portuguese mainland, Romania, Slovakia, Slovenia, South European Russia, Spanish mainland, Switzerland, Ukraine

Distribution in Turkey: Afyon, Ağrı, Amasya, Ankara, Antalya, Artvin, Aydın, Balıkesir, Bayburt, Bilecik, Bolu, Bursa, Çanakkale, Çorum, Edirne, Erzurum, Eskişehir, Gümüşhane, Hakkari, Hatay, İçel, Kahramanmaraş, Karabük, Kastamonu, Kırklareli, Kırşehir, Kütahya, Muğla, Nevşehir, Niğde, Ordu, Rize, Samsun, Sinop, Sivas, Tekirdağ, Tokat, Uşak, Van.

\section{Haematopota bigoti GOBERT, 1880}

Material examined: Keskin, $1150 \mathrm{~m}, 30.07 .2019,1$ q $q ; 1364 \mathrm{~m}, 08.07 .2020,2$ q ; $1700 \mathrm{~m}, 09.07 .2020,1$ \% ; Yahşihan, 1230 m, 16.07.2020, 1 우; Karakeçili, 931 m, 20.07.2020, 1 우.

Distribution worldwide: Austria, Britain I., Croatia, Danish mainland, European Turkey, French mainland, Germany, Hungary, Italian mainland, Near East, North Africa, Poland, Portuguese mainland, Romania, Slovakia, Spanish mainland, Sweden, The Netherlands, Former Yugoslavia.

Distribution in Turkey: Afyon, Ankara, Balıkesir, Çanakkale, Edirne, Elazı̆̆, Kars, Kırklareli, Konya, Kütahya, Tekirdağ, Yozgat.

\section{Haematopota hennauxi LECLERCQ, 1967}

Material examined: Keskin, 1150 m, 31.07.2019, 1 ㅇ; 1450 m, 08.07.2020, 2 q $\circ$; Sulakyurt, 1217 m, 06.08.2019, 1 우; $1300 \mathrm{~m}, 13.07 .2020,2$ 우; Balişeyh, 1275 m, 10.08.2019, 1 q ; ; 1385 m, 15.07.2020, 3 우; Yahşihan, 1252 m, 16.07.2020, 1 우; Karakeçili, $931 \mathrm{~m}, 20.07 .2020,3$ 우.

Distribution worldwide: Turkey.

Distribution in Turkey: Afyon, Ankara, Bolu, Çorum, Elazı̆̆, Eskişehir, Konya, Yozgat.

\section{Haematopota italica MEIGEN, 1804}

Material examined: Keskin, 1240 m, 29.07.2019, 1 q ; 08.07.2020, 3 q ; 1688 m, 09.07.2020, 1 q ; Delice, 1060 $\mathrm{m}, 03.08 .2019,1$ 우; $1278 \mathrm{~m}, 11.07 .2020,2$ 우; $1349 \mathrm{~m}, 12.07 .2020,1$ 우; Sulakyurt, 1324 m, 13.07.2020, 2 우; Balışeyh, 1581 m, 15.07.2020, 1 q q; Bahşili, 1122 m, 19.08.2019, 1 q ; 1530 m, 18.07.2020, 2 o ; Karakeçili, 931 m, 20.07.2020, 1 우우; Çelebi, 1426 m, 21.07.2020, 2 우우. 
Distribution worldwide: Albania, Austria, Belgium, Bosnia and Herzegovina, Bulgaria, Central European Russia, Croatia, Czech Republic, Danish mainland, East European Russia, Estonia, European Turkey, French mainland, Germany, Greek mainland, Hungary, Italian mainland, Liechtenstein, Lithuania, Luxembourg, Macedonia, Near East, Northwest European Russia, Poland, Portuguese mainland, Romania, Slovakia, South European Russia, Spanish mainland, Sweden, Switzerland, The Netherlands, Ukraine, Former Yugoslavia.

Distribution in Turkey: Adapazarı, Ankara, Balıkesir, Bartın, Bilecik, Bolu, Bursa, Çanakkale, Çankırı, Çorum, Diyarbakır, Düzce, Edirne, Elazı̆̆, Erzincan, Erzurum, Eskişehir, Gümüşhane, İçel, Karabük, Kastamonu, Kırklareli, Kütahya, Malatya, Muğla, Samsun, Sinop, Tokat, Trabzon, Zonguldak.

\section{Haematopota kemali SZILADY, 1923}

Material examined: Keskin, 1040 m, 29.07.2019, 1 우; 1280 m, 08.07.2020, 3 오; Delice, 1278 m, $11.07 .2020,2$ 우우 $1147 \mathrm{~m}, 26.08 .2020,1$ 우; Sulakyurt, $970 \mathrm{~m}, 06.08 .2019,2$ q $ᄋ$; $1224 \mathrm{~m}, 08.08 .2019,1$ 우; $1100 \mathrm{~m}, 13.07 .2020$, 3 우; Yahşihan, 862 m, 15.08.2019, 3 우; 1132 m, 18.08.2019, 1 o o ; 978 m, 16.07.2020, 4 o o ; Karakeçili, 791 m, 23.08.2019, 1 우; $931 \mathrm{~m}, 20.07 .2020,4$ 우.

Distribution worldwide: Turkey, Near East

Distribution in Turkey: Afyon, Ankara, Çankırı, Denizli, Elazı̆̆g, Erzurum, Eskişehir, İstanbul, Konya, Nevşehir, Niğde, Tekirdağ, Yozgat.

\section{Haematopota pallens LOEW, 1871}

Material examined: Delice, 1349 m, 02.08.2019, 1 q $q$; 1250 m, 11.07.2020, 2 q ; Balışeyh, 1275 m, $11.08 .2019,1$ 우우 $1710 \mathrm{~m}, 15.07 .2020,3$ 우우 $1315 \mathrm{~m}, 28.08 .2020,1$ 우; Bahşili, $1022 \mathrm{~m}, 20.08 .2019,1$ 우; $1522 \mathrm{~m}, 17.07 .2020$, 2 우우 Çelebi, $1426 \mathrm{~m}, 21.07 .2020,2$ 우; $1449 \mathrm{~m}, 22.07 .2020,1$ 우.

Distribution worldwide: Afghanistan, China, East Palaearctic, Moldova, Near East, Romania, South European Russia, Ukraine, Turkey

Distribution in Turkey: Afyon, Ankara, Balıkesir, Bilecik, Bursa, Diyarbakır, Elazığg, Eskişehir, İçel, İzmir, Kayseri Konya, Kütahya, Malatya, Muğla.

\section{Haematopota pluvialis (LINNEAUS, 1758)}

Material examined: Keskin, 1455 m, 01.08.2019, 2 우; 1620 m, 08.07.2020, 3 우; Yahşihan, 1016 m, 15.08.2019, 2 우우 $1215 \mathrm{~m}, 17.08 .2019,1$ 우; $1252 \mathrm{~m}, 16.07 .2020,3$ 우; Bahşili, $1139 \mathrm{~m}, 20.08 .2019,1$ 우; $1544 \mathrm{~m}, 17.07 .2020$, 2 우울 Çelebi, 1336 m, 21.07.2020, 1 우; Karakeçili, 931 m, 01.09.2020, 1 우우.

Distribution worldwide: Albania, Austria, Belgium, Bosnia and Herzegovina, Britain I., Bulgaria, Central European Russia, Croatia, Czech Republic, Danish mainland, East European Russia, East Palaearctic, Estonia, European Turkey, Finland, French mainland, Germany, Greek mainland, Hungary, Ireland, Italian mainland, Latvia, Liechtenstein, Lithuania, Luxembourg, Macedonia, Moldova, Near East, North European Russia, Northwest European Russia, Norwegian mainland, Poland, Portuguese mainland, Romania, Sardinia, Sicily, Slovakia, Slovenia, South European Russia, Spanish mainland, Sweden, Switzerland, The Netherlands, Ukraine, Former Yugoslavia.

Distribution in Turkey: Afyon, Ankara, Bilecik, Çanakkale, Edirne, ElazığEskişehir, İstanbul, Kayseri, Kırklareli, Konya, Niğde, Sivas, Tekirdağ, Yozgat.

\section{Haematopota sewelli AUSTEN, 1920}

Material examined: Keskin, 1046 m, 29.07.2019, 1 우; 1157 m, 31.07.2019, 1 우; 1240 m, 08.07.2020, 3 오; 1168 m, 25.08.2020, 2 우; Delice, 987 m, 02.08.2019, 4 우; $1201 \mathrm{~m}, 11.07 .2020,2$ q 웅 Sulakyurt, $1241 \mathrm{~m}, 07.08 .2019,3$ 우우; $966 \mathrm{~m}, 13.07 .2020,5$ 우; $1324 \mathrm{~m}, 27.08 .2020,1$ 우; Yahşihan, $900 \mathrm{~m}, 17.08 .2019,2$ 우; $1215 \mathrm{~m}, 16.07 .2020$, 3 우우 1066 m, 29.08.2020, 1 우; Karakeçili, 690 m, 23.08.2019, 1 우; 931 m, 20.07.2020, 5 o 우; Çelebi, 996 m, 21.07.2020, 2 우; $1126 \mathrm{~m}, 01.09 .2020,1$ 우.

Distribution worldwide: Israel, Iran, Turkey

Distribution in Turkey: Ankara, Çankırı, Elazığg, İzmir, Konya, Niğde, Trabzon Yozgat.

\section{Dasyramphis umbrinus (MEIGEN, 1820)}

Material examined: Keskin, $1150 \mathrm{~m}, 31.07 .2019,8$ 우 $1546 \mathrm{~m}, 01.08 .2019,2$ 우; $1367 \mathrm{~m}, 08.07 .2020,18$ q $ᄋ$; $1580 \mathrm{~m}, 09.07 .2020,14$ 우; Delice, $986 \mathrm{~m}, 05.08 .2019,1$ 우; $1150 \mathrm{~m}, 10.07 .2020,13$ 우; $1349 \mathrm{~m}, 12.07 .2020,10$ 우; Sulakyurt, $1324 \mathrm{~m}, 13.07 .2020,8$ 우; Yahşihan, $1217 \mathrm{~m}, 15.08 .2019,1$ 우; $1350 \mathrm{~m}, 16.07 .2020,9$ 우우 Karakeçili, 931 m, 20.07.2020, 4 o ㅇ; Çelebi, 996 m, 21.07.2020, 4 우; 1434 m, 22.07.2020, 11 우.

Distribution worldwide: Albania, Bosnia and Herzegovina, Bulgaria, Croatia, European Turkey, Greek mainland, Italian mainland, Macedonia, Near East, Romania, Slovenia, Former Yugoslavia,

Distribution in Turkey: Adana, Ankara, Antalya, Artvin, Balıkesir, Bolu, Bursa, Bursa, Çanakkale, Çankırı, Çorum, Denizli, Edirne, Erzincan, Erzurum, Eskişehir, Gümüşhane, Hakkari, İçel, İstanbul, İzmir, Kars, Kayseri, Kırklareli, Kırşehir, Konya, Kütahya, Manisa, Muğla, Muş, Nevşehir, Niğde, Osmaniye, Samsun, Sinop, Sivas, Uşak, Yalova, Yozgat. 


\section{Philipomyia aprica (MEIGEN, 1820)}

Material examined: Keskin, $1264 \mathrm{~m}, 29.07 .2019,6$ 우; $1574 \mathrm{~m}, 01.08 .2019,4$ 우; $1610 \mathrm{~m}, 08.07 .2020,16$ 우; 1255 m, 25.08.2020, 1 우; Delice, 1036 m, 03.08.2019, 2 우; 1278 m, 11.07.2020, 12 o ; 1349 m, $12.07 .2020,15$ o $\bigcirc$; Sulakyurt, 1130 m, 06.08.2019, 1 o ; 1063 m, 13.07.2020, 6 o ; 1324 m, 14.07.2020, 19 o $\circ$; Balışeyh, $1581 \mathrm{~m}$, 15.07.2020, 17 o o ; Yahşihan, 950 m, 14.08.2019, 4 oq ; 1250 m, 16.07.2020, 13 o o ; Bahşili, 1488 m, $17.07 .2020,9$ 우우; $1510 \mathrm{~m}, 19.07 .2020,16$ 우우 Çelebi, $1426 \mathrm{~m}, 21.07 .2020,5$ 우우.

Distribution worldwide: Albania, Austria, Belgium, Bosnia and Herzegovina, Bulgaria, Central European Russia, Croatia, Czech Republic, European Turkey, French mainland, Germany, Greek mainland, Hungary, Italian mainland, Liechtenstein, Macedonia, Near East, Poland, Portuguese mainland, Romania, Slovakia, Slovenia, Spanish mainland, Switzerland, Former Yugoslavia.

Distribution in Turkey: Afyon, Ankara, Antalya, Artvin, Balıkesir, Bayburt, Bingöl, Bolu, Bursa, Çanakkale, Erzurum, Eskişehir, Hakkari, İçel, İstanbul, Kars, Kastamonu, Kırklareli, Konya, Kütahya, Muş, Tekirdağ, Yalova.

Table 1. Species and numbers of horse flies collected in Kırıkkale during 2019 and 2020

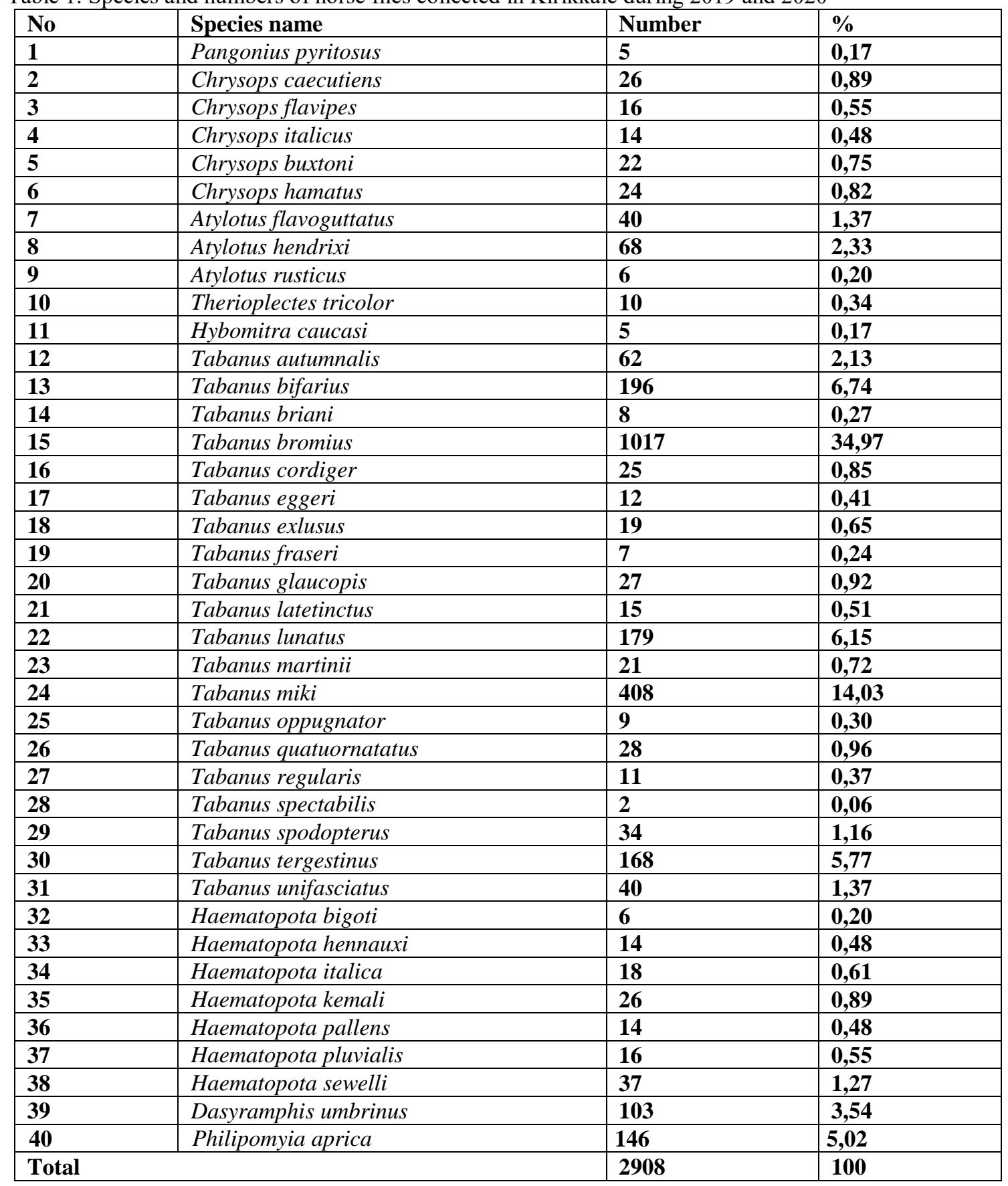




\section{Conclusions and discussion}

During the study total 40 horsefly species sampled and 36 species except Tabanus bromius Linneaus 1758; Tabanus regularis JAENNICKE, 1866; Tabanus spectabilis LOEW, 1858; Haematopota kemali SZILADY, 1923 first record for Kırıkkale. Pangonius (s. str.) pyritosus (Loew, 1859); Chrysops (s. str.) caecutiens (Linnaeus, 1758); Chrysops (s. str.) flavipes MEIGEN, 1804; Chrysops (s. str.) italicus MEIGEN, 1804; Chrysops (Petersenychrysops) buxtoni AUSTEN, 1922; Chrysops (Petersenychrysops) hamatus LOEW, 1858; Atylotus flavoguttatus (SZILADY, 1975); Atylotus hendrixi LECLERCQ, 1967; Atylotus rusticus (LINNAEUS, 1767); Therioplectes tricolor ZELLER, 1842; Hybomitra caucasi (SZILADY, 1923); Tabanus autumnalis LINNEAUS, 1761; Tabanus bifarius LOEW, 1858; Tabanus briani LECLERCQ, 1962; Tabanus cordiger MEIGEN, 1820; Tabanus eggeri SCHINERİ, 1868; Tabanus exlusus PANDELLE, 1883; Tabanus fraseri AUSTEN, 1925; Tabanus glaucopis MEIGEN, 1936; Tabanus latetinctus AUSTEN, 1920; Tabanus lunatus FABRICIUS, 1794; Tabanus martinii KRÖBER, 1928; Tabanus miki BRAUER, 1880; Tabanus oppugnator AUSTEN, 1925; Tabanus quatuornatatus MEIGEN, 1820; Tabanus spodopterus MEIGEN, 1820; Tabanus tergestinus EGGER, 1859; Tabanus unifasciatus LOEW, 1858; Haematopota bigoti GOBERT, 1881; Haematopota hennauxi LECLERCQ, 1967; Haematopota italica MEIGEN, 1804; Haematopota pallens LOEW, 1870; Haematopota pluvialis (LINNEAUS, 1761); Haematopota sewelli AUSTEN, 1920; Dasyramphis umbrinus (MEIGEN, 1820) and Philipomyia aprica (MEIGEN, 1820) are reported for the first time from study area.

$\% 34,97$ adult specimens belong to Tabanus bromius is the most abundant and the most common species like similar studies [4, 18]. Geographical conditions, temperature and seasonal factors provide dense distribution of the $T$. bromius in Kırıkkale. Tabanus miki \%14,03; Tabanus bifarius \%6,74; Tabanus lunatus \%6,15 and Tabanus tergestinus $\% 5,77$ followed the Tabanus bromius with species richness (Table 1).

Total collected specimen distribution as percentage; \%56,39 in July, \%38,78 in August and \%4,84 in September. This is similar to the present studies about seasonal activities of horseflies. In Turkey horsefly activities begin in the middle of May and continue to the middle of September [15, 19, 23-26, 28]. Unfortunately in May and June field studies could not be carried out because of the Covid-19 pandemic which caused the travel limitation. However specimens collected at the maximum activity periods of horseflies.

The collection localities are especially selected at the rural areas where livestock and animal husbandry are dense. Due to female tabanids are known as mechanical vectors of viruses, bacteria, protozoans and helminths that cause various diseases in wild and domestic animals [8, 12, 31], present study is also important for avoiding the spread of these diseases via Tabanids. The results of this study involve the whole tabanids distribution in the Kirikkale. These faunistic results contribute information of the Tabanidae in Turkey and also provide prediction of probability of losses in livestock production.

\section{Acknowledgements} 19ADP018).

This study was supported by the Research Foundation of Eskişehir Technical University (Project Number:

\section{Kaynaklar}

[1] Altunsoy, F. (2018). New records for the horse fly (Diptera: Tabanidae) fauna of Turkey and description of Hybomitra tanatmisi sp. nov. Türkiye Entomoloji Dergisi, 42(2), 93-108.

[2] Altunsoy, F., \& Kilıç, A. Y. (2010). A new record for Turkish Tabanidae (Insecta: Diptera) fauna. Journal of Entomological Research Society, 12(2), 109-111.

[3] Altunsoy, F., \& Kılıç, A. Y. (2011). New data about Tabanus karaosus Timmer, 1984 (Diptera: Tabanidae) from Turkey. Journal of Entomological Research Society, 13(1), 75-80.

[4] Altunsoy, F., \& Kılıç, A. Y. (2012). Seasonal Abundance of Horse Fly Diptera Tabanidae in Western Anatolia. Journal of Entomological Research Society, 14(1), 95-105.

[5] Altunsoy, F., Kılıç, A. Y., \& Gören, T. (2010). Düzce İli Tabanidae (Diptera: Insecta) faunası. Sakarya Üniversitesi Fen Bilimleri Dergisi, 14(1), 29-32.

[6] Barros, A. M. (2001). Seasonality and relative abundance of Tabanidae (Diptera) captured on horses in the Pantanal, Brazil. Memorias do Instituto Oswaldo Cruz(96), 917-923.

[7] Büber, H., Kılıç, A. Y., \& Altunsoy, F. (2011). Afyon ili Tabanidae (Insecta: Diptera) faunasına katkılar. Afyon Kocatepe University Journal of Science, 9(2), 43-50.

[8] Chippaux, J. P., Bouchite, B., Demanou, M., Moralis, I., \& Le Goff, G. (2000). Density and dispersal of the loaiasis vector Chrysops dimidiata in southern Cameroon. Medical and Veterinary Entomology(14), 339-344. 
[9] Chvala, M. (1988). Family Tabanidae. A. Soos, \& A. Papp içinde, Catalogue of Palaearctic Diptera (Cilt 5, s. 7197). Amsterdam, The Netherlands: Elsevier Science Publishers.

[10] Chvala, M., Lyneborg, L., \& Moucha, J. (1972). The horse flies of Europe (Diptera: Tabanidae). Hampton: E. W. Classey Ltd.

[11] Coscaron, S., \& Martinez, M. (2019). Checklist of Tabanidae (Insecta: Diptera) from Uruguay. Journal of Entomological Society Argentina(78), 40-46.

[12] Desquesnes, M., \& Dia, M. L. (2004). Mechanical transmission of Trypanosoma vivax in cattle by the African tabanid Atylotus fuscipes. Veterinary Parasitology(14), 9-19.

[13] Goldblatt, P., \& Manning, J. C. (2000, September). Champion Pollinators. Long-proboscid Flies in a Biological System Unique to Southern Africa. Veld and Flora, 118-121.

[14] Hayat, R., \& Özbek, H. (1992). Doğu Anadolu Bölgesi Tabanidae (Diptera) türleri üzerine faunistik çalışmalar. Türkiye II. Entomoloji Kongresi Bildirileri No 5, (s. 637-646). Adana, Turkey: Entomoloji Derneği Yayınları.

[15] Kılıç, A. Y. (1993). Eskişehir ve Çevresi Tabanus bromius L., T. exlusus Pand., T. glaucopis Meig., T. spodopterus ponticus Ols., Mch., Chv., ve Philipomyia aprica (Meig) (Diptera: Tabanidae) türlerinin mevsimsel aktiviteleri üzerine bir çalışma. Doğa Türk Zooloji Dergisi, 17(3), 303-310.

[16] Kılıç, A. Y. (2005). ). Bartın, Karabük ve Zonguldak illeri Tabanidae (Diptera) faunası üzerinde araştırmalar. Türliye Entomoloji Dergisi, 29(2), 51-60.

[17] Kılıç, A. Y. (2006). New additions and errata to the checklist of Tabanidae (Insecta: Diptera) fauna of Turkey. Turkish Journal of Zoology, 2(30), 335-343.

[18] Kılıç, A. Y., \& Altunsoy, F. (2011). Kastamonu ili Tabanidae (Insecta: Diptera) Faunasına Katkılar. Kastamonu Üniversitesi Orman Fakültesi Dergisi, 11(1), 1-8.

[19] Kılıç, A. Y., \& Öztürk, R. (2002). Sultandağı çevresinin Tabanidae (Diptera) Faunası üzerine çalışmalar. Anadolu Üniversitesi Bilim ve Teknoloji Dergisi, 3(2), 307-316.

[20] Kılıç, A. Y., \& Schacht, W. (1995). Eine neue Bremsenart aus der West-Türkei (Diptera: Tabanidae). Entomofauna Band.,, 16(10), 245-252.

[21] Kılıç, A. Y., Altunsoy, F., \& Afacan, Y. (2014). New records for Turkish Tabanidae (Insecta: Diptera) fauna. Türkiye Entomoloji Dergisi, 38(3), 245-253.

[22] Krinsky, W. L. (1976). Animal disease agents transmitted by horse flies and deer flies (Diptera: Tabanidae). Journal of Medical Entomology(13), 225-275.

[23] Leclercq, M. (1966a). Revision systematique et biogeographique des Tabanidae (Diptera) Palearctiques, Tabaninae (Cilt 79). Mem. Ins. Roy. Sci. Nat. Belg. II.

[24] Leclercq, M. (1966b). Tabanidae (Diptera) de Turquie diagnosis d'Atylotus hendrixi, Haematopota coolsi, Haematopota delozi n. spp. Bull. Rech. Agron. Gembloux, 1(3), 463-477.

[25] Leclercq, M. (1967a). Tabanidae (Diptera) de Turquie, II. Diagnosis D'Hybomitra okayi, Atylotus hendrixi, et Haematopota hennauxi n. spp. Bull. Rech. Agron. Gembloux, 2(1), 106-127.

[26] Leclercq, M. (1967b). Tabanidae (Diptera) de Turquie III. Bull. Rech. Agron. Gembloux, 2(4), 707-710.

[27] Olsufjev, N. G. (1977). The Fauna of USSR Diptera, VII, 2: Tabanidae (Faune de 1'URSS insectes Dipteres, VII, 2: Tabanidae). . Moscow, Leningrad, Russia: Institute of the Russian Academy of Sciences (Travaux de l'Institut zoologique de l'Academie des Sciences des l'URSS) New Series No. 113.

[28] Parvu, C., \& Giray, H. (1984). Contribution to the knowledge of some Tabanids (Diptera) of Turkey. Travaux du Museum National d'Histoire Naturelle "Grigore Antipa", 25, 217-225.

[29] Ren, D. (1998). Flower-associated Brachycera flies as Fossil Evidence for Jurassic Angiosperm Origins. Science(280), 85-88.

[30] Schacht, W. (1987). Ein weiterer Beitrag bremsen fauna der Türkei (Diptera: Tabanidae). Entomofauna, 8(33), 485-496.

[31] Votypka, J., Brzonova , J., Jezek, J., \& Modry, D. (2019, September). Horseflies (Diptera: Tabanidae) of three West African countries: A faunistic update, barcoding analysis and trypanosome occurrence. Acta Tropica(197).

[32] Yücel, Ş. (1987). İç Anadolu Bölgesinde bulunan Tabanidae (Diptera) türleri üzerinde araştırmalar. Ankara Üniversitesi Sağlık Bilimleri Enstitüsü. 